PROCEEDINGS OF THE

AMERICAN MATHEMATICAL SOCIETY

Volume 135, Number 10, October 2007, Pages 3283-3293

S 0002-9939(07)08856-9

Article electronically published on June 20, 2007

\title{
THE LIMITING CASE OF THE MARCINKIEWICZ INTEGRAL: GROWTH FOR CONVEX SETS
}

\author{
N. KRUGLYAK AND E. A. KUZNETSOV \\ (Communicated by N. Tomczak-Jaegermann)
}

AbStract. The Marcinkiewicz integral

$$
I_{\lambda}(x)=\int_{\Omega} \frac{\left(\text { dist }\left(y, \mathbb{R}^{n} \backslash \Omega\right)\right)^{\lambda}}{|x-y|^{n+\lambda}} d y, \text { where } \lambda>0,
$$

plays a well-known and prominent role in harmonic analysis. In this paper, we estimate the growth of it in the limiting case $\lambda \rightarrow 0$. Throughout, we assume that $\Omega$ is convex; it is interesting that this condition cannot be dropped.

\section{INTRODUCTION}

Our aim in this paper is to prove the following theorem:

Theorem 1. Let $\Omega$ be a bounded open convex subset of $\mathbb{R}^{n}$. For $x \in \mathbb{R}^{n} \backslash \Omega$, put

$$
F_{\Omega}(x)=\int_{\Omega} \frac{1}{|x-y|^{n}} d y .
$$

Let $\Omega_{t}=\left\{x \in \mathbb{R}^{n} \mid \operatorname{dist}(x, \Omega)<t\right\}$. Then

$$
\sup _{x \in \mathbb{R}^{n} \backslash \Omega_{t}} F_{\Omega}(x) \leq c(n) \frac{|\Omega|}{\left|\Omega_{t} \backslash \Omega\right|},
$$

where $c=c(n)>0$ is a constant dependent only on the dimension $n$.

The most interesting feature of this result is that the constant in (1.2) is independent of the geometry of the convex set $\Omega$. It should be noted that the convexity of $\Omega$ is essential. In $\S 4$ we present an example of a bounded domain $\Omega$ for which the constant $c$ in (1.2) is infinite. It is also possible to construct a bounded domain $\Omega$ with a smooth boundary and with an arbitrarily large constant $c$ in (1.2). Moreover, the estimate (1.2) is sharp in the sense that both sides of it are equivalent to $t^{-n}$ for large $t$.

Theorem 1 will be proved in $\S \S 2-3$; here we comment on the question of why the function (1.1) is interesting.

Received by the editors May 18, 2006 and, in revised form, July 13, 2006.

2000 Mathematics Subject Classification. Primary 42B20, 42B25.

Key words and phrases. Riesz potential, Marcinkiewicz integral, Poisson's equation.

(C)2007 American Mathematical Society Reverts to public domain 28 years from publication 
First of all this function can be considered as a bordering case between the Marcinkiewicz integral

$$
I_{\lambda}(x)=\int_{\Omega} \frac{\left(\operatorname{dist}\left(y, \mathbb{R}^{n} \backslash \Omega\right)\right)^{\lambda}}{|x-y|^{n+\lambda}} d y, \quad \text { where } \lambda>0,
$$

and the Riesz potential operator

$$
R(f)(x)=\int_{\mathbb{R}^{n}} \frac{f(y)}{|x-y|^{n-\alpha}} d y, \quad \alpha>0
$$

for $f=\chi_{\Omega}$.

Moreover, the function (1.1) arises naturally in estimates of Poisson integral (see [8]), the moment problem, operator theory and computerized tomography (see, for example, the survey [7] and [1], 3]). It is also connected with an analytic continuation of Cauchy transform, free boundary problems and with fluid mechanics (Hele-Shaw flow) (see [2]).

The authors are very thankful to Professors Lars Inge Hedberg, Börje Nilsson and Peter Sjögren for fruitful discussions.

\section{Auxiliary lemmas}

In this section we prove some statements to be used subsequently.

\subsection{Independence of a metric in $\mathbb{R}^{n}$. Put}

$$
\varphi(t)=\sup _{x \in \mathbb{R}^{n} \backslash \Omega_{t}} F_{\Omega}(x), \text { for } t>0 .
$$

In the definition $\Omega_{t}=\left\{x \in \mathbb{R}^{n} \mid \operatorname{dist}(x, \Omega)<t\right\}$ of $\Omega_{t}$ we assume that

$$
\operatorname{dist}(x, \Omega)=\inf _{y \in \Omega}\left(\sum_{i=1}^{n}\left(x_{i}-y_{i}\right)^{2}\right)^{\frac{1}{2}} \text {. }
$$

In what follows, it will be convenient to use the metric

$$
\rho(x, y)=\max _{i=1, \ldots, n}\left|x_{i}-y_{i}\right|
$$

in place of the Euclidean metric in $\mathbb{R}^{n}$. Consequently, (2.2) is replaced by

$$
\rho(x, \Omega)=\inf _{y \in \Omega} \rho(x, y) .
$$

We show that it suffices to prove the version of Theorem 1 in which the distance (2.4) is used in place of (2.2) throughout, i.e., $\Omega_{t}$ and $\varphi(t)$ are replaced by

$$
\begin{gathered}
\Omega_{t, \rho}=\left\{x \in \mathbb{R}^{n} \mid \rho(x, \Omega)<t\right\}, \\
\varphi_{\rho}(t)=\sup _{x \in \mathbb{R}^{n} \backslash \Omega_{t, \rho}} F_{\Omega}(x) .
\end{gathered}
$$

Indeed, all norms on $\mathbb{R}^{n}$ are equivalent, therefore

$$
\Omega_{c_{1}, \rho} \subset \Omega_{t} \subset \Omega_{c_{2} t, \rho}
$$

with constants $c_{2}>c_{1}>0$ independent of $t>0$. So

$$
\varphi_{\rho}\left(c_{2} t\right) \leq \varphi(t) \leq \varphi_{\rho}\left(c_{1} t\right) .
$$


If Theorem 1 is proved with the metric $\rho$, then

$$
\varphi(t)=\sup _{x \in \mathbb{R}^{n} \backslash \Omega_{t}} F_{\Omega}(x) \leq \sup _{x \in \mathbb{R}^{n} \backslash \Omega_{c_{1} t, \rho}} F_{\Omega}(x) \leq c(n) \frac{|\Omega|}{\left|\Omega_{c_{1} t, \rho} \backslash \Omega\right|} \leq c(n) \frac{|\Omega|}{\left|\Omega_{\frac{c_{1}}{c_{2}} t} \backslash \Omega\right|} .
$$

Consequently, we only need to prove the inequality

$$
\left|\Omega_{\lambda t} \backslash \Omega\right| \geq c\left|\Omega_{t} \backslash \Omega\right|
$$

with a constant $c=c(n, \lambda)>0$ independent of $t$. If $\lambda \geq 1$, then $\Omega_{\lambda t} \supset \Omega_{t}$ and we can take $c=1$, so we can assume that $\lambda<1$.

To prove this, take $x \in \Omega_{t} \backslash \Omega$. Then there exists $y \in \Omega$ such that the ball $B(y, t)$ of radius $t$ and centered at $y$ contains $x$. Since $\Omega$ is open, there is no loss of generality in assuming that $y \in \partial \Omega\left(\partial \Omega\right.$ is the boundary of $\Omega$ ). Therefore, $\Omega_{t} \backslash \Omega$ is covered by certain balls of radius $t$ whose centers lie on $\partial \Omega$. By the Wiener covering theorem (see [9, p. 9] or the so-called $5 r$ theorem in [6]), we can find a finite collection $y_{1}, \ldots, y_{N} \in \partial \Omega$ such that the balls $B\left(y_{i}, t\right)$ are mutually disjoint and the balls $B\left(y_{i}, 5 t\right)$ cover $\Omega_{t} \backslash \Omega$. Therefore,

$$
\left|\Omega_{t} \backslash \Omega\right| \leq 5^{n} w_{n} \cdot(t)^{n} \cdot N,
$$

where $w_{n}$ is the volume of the $n$-dimensional unit ball.

Now, consider the balls $B\left(y_{i}, \lambda t\right)$. For each $i$, a support hyperplane $\pi_{i}$ to the convex set $\Omega$ passes through $y_{i}$, and $\Omega$ lies entirely in only one of the two halfspaces determined by $\pi_{i}$. The intersection of $B\left(y_{i}, \lambda t\right)$ with the other half-space is included in $\Omega_{t} \backslash \Omega$, and the balls $B\left(y_{i}, \lambda t\right)$ are mutually disjoint (because so are the balls $B\left(y_{i}, t\right)$, and $\left.\lambda<1\right)$. It follows that

$$
\left|\Omega_{\lambda t} \backslash \Omega\right| \geq \frac{1}{2} \lambda^{n} w_{n} t^{n} N .
$$

Combining this with (2.10), we obtain

$$
\left|\Omega_{\lambda t} \backslash \Omega\right| \geq \frac{\lambda^{n}}{2 \cdot 5^{n}}\left|\Omega_{t} \backslash \Omega\right| .
$$

This proves (2.9).

In fact, the above argument shows that it suffices to prove Theorem 1 for any particular metric generated by some norm in $\mathbb{R}^{n}$.

2.2. Reduction to the case of an $n$-dimensional rectangle. In this subsection we show that it suffices to prove Theorem 1 only for the rectangle $\left(-a_{1}, a_{1}\right) \times \cdots \times$ $\left(-a_{n}, a_{n}\right)$ in the role of $\Omega$.

Indeed, let $\Omega$ be an arbitrary open and bounded convex set. By the John theorem, there exists an ellipsoid $E$ with $\Omega \subset E$ and $|E| \leq c(n)|\Omega|$. Since translations and orthogonal transformations change neither distances nor volumes (consequently, nor the values of $F_{\Omega}$ ), we may assume that the center of $E$ is at zero and its axes are directed along coordinate axes. Let $a_{i}$ be the length of the $i$ th semiaxis of $E$. Then for the rectangle

$$
R=\left(-a_{1}, a_{1}\right) \times \cdots \times\left(-a_{n}, a_{n}\right)
$$

we have $\Omega \subset R$ and

$$
|R| \leq c(n)|\Omega| .
$$

Now, suppose that Theorem 1 is true for $R$. We show that then it is true for $\Omega$. 
Indeed, since $\Omega \subset R$ and $\Omega$ and $R$ are bounded and convex, we have

$$
\left|\Omega_{t} \backslash \Omega\right| \leq\left|R_{t} \backslash R\right|
$$

(see [4). Put

$$
\varphi_{R}(t)=\sup _{x \in \mathbb{R}^{n} \backslash R_{t}} F_{R}(x) .
$$

It suffices to prove the estimate

$$
\varphi(t) \leq c(n) \varphi_{R}(\lambda t),
$$

with a constant $\lambda=\lambda(n)$ depending only on $n$.

Indeed, if Theorem 1 is true for $R$, then (2.14) and (2.13) imply 1

$$
\varphi_{R}(t) \leq c(n) \frac{|R|}{\left|R_{t} \backslash R\right|} \leq c(n) \frac{|\Omega|}{\left|R_{t} \backslash R\right|} \leq c(n) \frac{|\Omega|}{\left|\Omega_{t} \backslash \Omega\right|} .
$$

Consequently, if we prove (2.15), then from (2.9) we obtain

$$
\varphi(t) \leq c(n) \varphi_{R}(\lambda t) \leq c(n) \frac{|\Omega|}{\left|\Omega_{\lambda t} \backslash \Omega\right|} \leq c(n) \frac{|\Omega|}{\left|\Omega_{t} \backslash \Omega\right|} .
$$

Inequality (2.8) shows that it suffices to prove (2.15) in the case of the metric $\rho(x, y)=\max _{i=1, \ldots, n}\left|x_{i}-y_{i}\right|$, i.e., under the assumption that the sets $\Omega_{t}, R_{t}$ and the functions $\varphi, \varphi_{R}$ are defined on the basis of the metric $\rho$.

To do this, we consider a point $x \in \mathbb{R}^{n} \backslash \Omega_{t}$, i.e., $\rho(x, \Omega) \geq t$, and estimate the value $F_{\Omega}(x)$. Let $y \in \partial \Omega$ be a point nearest to $x$, i.e., $\rho(x, y)=\rho(x, \Omega)$. Let $\sigma_{1}, \sigma_{2}, \ldots, \sigma_{2^{n}}$ denote the vertices of the rectangle $\bar{R}=\left[-a_{1}, a_{1}\right] \times \cdots \times\left[-a_{n}, a_{n}\right]$, and let $\bar{R}_{i}$ be the translate of $\bar{R}$ such that $\sigma_{i}$ is translated to $y$. Since $y \in \bar{R}$, it is easily seen that $\bar{R} \subset \bigcup_{i} \bar{R}_{i}$ and, consequently,

$$
\Omega \subset \bigcup_{i} \bar{R}_{i} .
$$

Among the $\bar{R}_{i}$, we select those that intersect $\Omega$, i.e., we consider the set $I=$ $\left\{i \mid \Omega \cap \bar{R}_{i} \neq \emptyset\right\}$. Then (2.16) and the definition of $F_{\Omega}(x)$ imply

$$
F_{\Omega}(x) \leq \sum_{i \in I} F_{R_{i}}(x) .
$$

If we show that $\rho\left(x, R_{i}\right) \geq t$ for $i \in I$, then we obtain

$$
F_{\Omega}(x) \leq \sum_{i \in I} \varphi_{R_{i}}(t) \leq|I| \varphi_{R}(t) \leq 2^{n} \varphi_{R}(t)
$$

and, consequently,

$$
\varphi(t) \leq 2^{n} \varphi_{R}(t) .
$$

Thus, it remains to prove that the relations $\rho(x, \Omega)=\rho(x, y) \geq t$ imply $\rho\left(x, R_{i}\right)$ $\geq t$ for all $i \in I$. Suppose the contrary, i.e., there is $z \in R_{i}(i \in I)$ with $\rho(x, z)<t$. Now, we observe that all points $u$ lying in $R_{i}$ near $y$ can be written in the form

$$
\begin{aligned}
u & =\left(y_{1}, y_{2}, \ldots, y_{n}\right)+\left(\alpha_{1}\left(z_{1}-y_{1}\right), \ldots, \alpha_{n}\left(z_{n}-y_{n}\right)\right) \\
& =\left(\alpha_{1} z_{1}+\left(1-\alpha_{1}\right) y_{1}, \ldots, \alpha_{n} z_{n}+\left(1-\alpha_{n}\right) y_{n}\right)
\end{aligned}
$$

\footnotetext{
${ }^{1}$ Here and below, the notation $c(n)$ is used for constants depending only on the dimension $n$; their particular values may vary from one occurrence of this symbol to another.
} 
with $\alpha_{1}, \ldots, \alpha_{n} \in(0,1)$. Since $\rho(x, z)<t \leq \rho(x, y)$, we have

$$
\begin{gathered}
\rho(x, u)=\max _{i}\left|x_{i}-\alpha_{i} z_{i}-\left(1-\alpha_{i}\right) y_{i}\right| \\
\leq \max _{i}\left(\alpha_{i}\left|x_{i}-z_{i}\right|+\left(1-\alpha_{i}\right)\left|x_{i}-y_{i}\right|\right)<\rho(x, y) .
\end{gathered}
$$

Next, since $\Omega \cap R_{i} \neq \emptyset$ and $y \in \bar{\Omega}$, by the convexity of $\Omega$ we see that in $\Omega \cap R_{i}$ there are points $u$ arbitrarily close to $y$. Then $\rho(x, u)<\rho(x, y)$ by (2.18), contrary to the choice of $y$.

The contradiction proves the claim.

\section{Proof of THEOREM 1}

IN THE CASE WHEN $\Omega$ IS AN $n$-DIMENSIONAL RECTANGLE

We saw in $\S 2$ that it suffices to prove the inequality

$$
\varphi(t)=\sup _{x \in \mathbb{R}^{n} \backslash \Omega_{t}} F_{\Omega}(x) \leq c(n) \frac{|\Omega|}{\left|\Omega_{t} \backslash \Omega\right|},
$$

where

$$
\begin{gathered}
\Omega=\left(-a_{1}, a_{1}\right) \times \cdots \times\left(-a_{n}, a_{n}\right), \\
\Omega_{t}=\left\{x \in \mathbb{R}^{n} \mid \rho(x, \Omega)<t\right\},
\end{gathered}
$$

and

$$
\rho(x, y)=\max \left|x_{i}-y_{i}\right| \text {, where } x=\left(x_{1}, \ldots, x_{n}\right) \text { and } y=\left(y_{1}, \ldots, y_{n}\right) .
$$

In this section we verify (3.1). The proof is based on two lemmas. Below we assume for definiteness that

$$
0<a_{1} \leq \cdots \leq a_{n} .
$$

Lemma 1. a) If $0<t<a_{1}$, then

$$
\left|\Omega_{t} \backslash \Omega\right| \leq c(n) \frac{t}{a_{1}}|\Omega|,
$$

b) If $a_{k} \leq t \leq a_{k+1}(k=1, \ldots, n-1)$ or $t \geq a_{n}(k=n)$, then

$$
\left|\Omega_{t} \backslash \Omega\right| \leq c(n) \frac{t}{a_{1}} \cdot \ldots \cdot \frac{t}{a_{k}}|\Omega| .
$$

Proof. Inequality (3.6) is a consequence of the estimates

$$
\begin{aligned}
& \left|\Omega_{t} \backslash \Omega\right| \leq\left(2 a_{1}+2 t\right) \cdots\left(2 a_{n}+2 t\right)-2 a_{1} \cdots 2 a_{n} \\
& =2 a_{1} \cdots 2 a_{n}\left[\left(1+\frac{t}{a_{1}}\right) \cdots\left(1+\frac{t}{a_{n}}\right)-1\right] \\
& \quad \leq|\Omega|\left[\left(1+\frac{t}{a_{1}}\right)^{n}-1\right] \leq n \cdot 2^{n-1} \cdot \frac{t}{a_{1}}|\Omega|,
\end{aligned}
$$

where at the last step we have used the formula $f(b)-f(a)=f^{\prime}(c) \cdot(b-a)$ for the function $f(x)=(1+x)^{n}$ and $a=0, b=\frac{t}{a_{1}}$.

Inequality (3.7) is also elementary:

$$
\begin{gathered}
\left|\Omega_{t} \backslash \Omega\right| \leq\left(2 a_{1}+2 t\right) \cdots\left(2 a_{n}+2 t\right) \leq(4 t)^{k}\left(4 a_{k+1}\right) \cdot \ldots \cdot\left(4 a_{n}\right) \\
=2^{n} \frac{t}{a_{1}} \cdot \ldots \cdot \frac{t}{a_{k}}|\Omega| .
\end{gathered}
$$


In the proof of another lemma, we shall use the fact that it suffices to estimate $\sup _{x \in \mathbb{R}^{n} \backslash \Omega_{t}} F_{\Omega}(x)$ for $x$ belonging to the union of coordinate axes. Moreover, the following statement is true.

Proposition 1. For $\Omega=\left(-a_{1}, a_{1}\right) \times \cdots \times\left(-a_{n}, a_{n}\right)$ we have

$$
\sup _{x \in \mathbb{R}^{n} \backslash \Omega_{t}} F_{\Omega}(x) \leq c(n) \max _{i=1, \ldots, n} F_{\Omega}\left(\left(a_{i}+t\right) e_{i}\right),
$$

where $\left\{e_{1}, \ldots, e_{n}\right\}$ is the standard basis in $\mathbb{R}^{n}$.

Proof. Suppose $x \in \mathbb{R}^{n} \backslash \Omega_{t}$, i.e., $\rho(x, \Omega) \geq t$. The symmetry of $\Omega$ allows us to assume that all coordinates of $x$ are nonnegative. Then

$$
\rho(x, \Omega)=\max _{i}\left(x_{i}-a_{i}\right) \geq t .
$$

There is an index $i_{0}$ with $x_{i_{0}}-a_{i_{0}}=\rho(x, \Omega)$. We replace by $a_{i}$ all coordinates $x_{i}$ of $x$ such that $i \neq i_{0}$ and $x_{i}-a_{i}>0$, obtaining a new vector $\tilde{x}=\left(\tilde{x}_{1}, \ldots, \tilde{x}_{n}\right)$ with the property

$$
|\tilde{x}-y| \leq|x-y| \text {, for any } y \in \Omega \text {. }
$$

Then the relation $\rho(\tilde{x}, \Omega)=\rho(x, \Omega)$ and (1.1) show that $F_{\Omega}(\tilde{x}) \geq F_{\Omega}(x)$. Thus, when estimating the left-hand side of (3.8), we may assume that exactly one coordinate $x_{i_{0}}$ of $x$ is greater than $a_{i_{0}}$.

Next, consider the translation

$$
\begin{aligned}
\tilde{y}_{i} & =y_{i}-x_{i}, \text { for all } i \neq i_{0}, \\
\tilde{y}_{i_{0}} & =y_{i_{0}},
\end{aligned}
$$

which takes $x$ to $x_{i_{0}} e_{i_{0}}$, and $\Omega$ to some rectangle $\tilde{\Omega}$. Clearly, $F_{\Omega}(x)=F_{\tilde{\Omega}}\left(x_{i_{0}} e_{i_{0}}\right)$. Furthermore, the set

$$
\bar{\Omega}=\left(-2 a_{1}, 2 a_{1}\right) \times \cdots \times\left(-a_{i_{0}}, a_{i_{0}}\right) \times \cdots \times\left(-2 a_{n}, 2 a_{n}\right)
$$

(we multiply all $a_{i}$ except $a_{i_{0}}$ by 2 ) contains $\tilde{\Omega}$, whence it follows that $F_{\Omega}(x) \leq$ $F_{\bar{\Omega}}\left(x_{i_{0}} e_{i_{0}}\right)$. The change of variables

$$
\begin{aligned}
\bar{y}_{i} & =\frac{1}{2} \tilde{y}_{i}, \text { for all } i \neq i_{0}, \\
\bar{y}_{i_{0}} & =y_{i_{0}},
\end{aligned}
$$

maps $\bar{\Omega}$ into $\Omega$ and fixes $x_{i_{0}} e_{i_{0}}$. After simple calculations this yields

$$
F_{\bar{\Omega}}\left(x_{i_{0}} e_{i_{0}}\right) \leq 2^{n-1} F_{\Omega}\left(x_{i_{0}} e_{i_{0}}\right) .
$$

Therefore, $F_{\Omega} \leq 2^{n-1} F_{\Omega}\left(x_{i_{0}} e_{i_{0}}\right)$. It remains to observe that $F_{\Omega}\left(x_{i_{0}} e_{i_{0}}\right)$ increases as $x_{i_{0}}$ decreases, and so, under the condition $\rho\left(x_{i_{0}} e_{i_{0}}, \Omega\right) \geq t$, its minimal value is attained for $x_{i_{0}}=a_{i_{0}}+t$.

We shall use the following elementary estimates:

$$
\begin{aligned}
& \int_{t}^{t+a} \frac{1}{s^{m}} d s \leq \int_{t}^{t+a} \frac{1}{t^{m}} d s=\frac{a}{t^{m}}, \text { where } t, a>0 \text { and } m \geq 0 \\
& \int_{t}^{t+a} \frac{1}{s^{m}} d s \leq \int_{t}^{\infty} \frac{1}{s^{m}} d s=\frac{1}{m-1} \cdot \frac{1}{t^{m-1}}, \text { where } t, a>0 \text { and } m>1 .
\end{aligned}
$$


Also, we need the fact that the integrals

$$
c_{m, n}=\int_{\mathbb{R}^{m}} \frac{1}{\left(1+\sum_{i=1}^{m} u_{i}^{2}\right)^{\frac{n}{2}}} d u_{1} \cdots d u_{m}<\infty
$$

are finite for $m<n$.

Lemma 2. a) If $0<t<a_{1}$, then

$$
\sup _{x \in \mathbb{R}^{n} \backslash \Omega_{t}} F_{\Omega}(x) \leq c(n) \frac{a_{1}}{t} .
$$

b) If $a_{k} \leq t \leq a_{k+1}(k=1, \ldots, n-1)$ or $t \geq a_{n}(k=n)$, then

$$
\sup _{x \in \mathbb{R}^{n} \backslash \Omega_{t}} F_{\Omega}(x) \leq c(n) \frac{a_{1}}{t} \cdot \ldots \cdot \frac{a_{k}}{t},
$$

where $c(n)$ is a constant depending on the dimension $n$ only.

Proof. We start with case $a)$. By Proposition1, we may assume that $x=\left(t+a_{i_{0}}\right) e_{i_{0}}$. We consider two cases: $i_{0}=1$ and $i_{0} \neq 1$. In the first case

$$
F_{\Omega}(x)=\int_{\Omega} \frac{1}{\left(\left(t+a_{1}-y_{1}\right)^{2}+\sum_{i \neq 1} y_{i}^{2}\right)^{\frac{n}{2}}} d y .
$$

We substitute $s=t+a_{1}-y_{1}$ and extend integration in $y_{2}, \ldots, y_{n}$ over $(-\infty,+\infty)$, obtaining

$$
F_{\Omega}(x) \leq \int_{t}^{t+2 a_{1}}\left(\int_{\mathbb{R}^{n-1}} \frac{1}{\left(s^{2}+\sum_{i \neq 1} y_{i}^{2}\right)^{\frac{n}{2}}} d y_{2} \cdots d y_{n}\right) d s .
$$

Factoring out $s^{n}$ in the denominator and changing the variables $u_{2}=\frac{y_{2}}{s}, \ldots, u_{n}=$ $\frac{y_{n}}{s}$, we arrive at

$$
F_{\Omega}(x) \leq \int_{t}^{t+2 a_{1}} \frac{1}{s}\left(\int_{\mathbb{R}^{n-1}} \frac{1}{\left(1+\sum_{i \neq 1} u_{i}^{2}\right)^{\frac{n}{2}}} d u_{2} \cdots d u_{n}\right) d s .
$$

Therefore, (3.9) and (3.11) imply

$$
F_{\Omega}(x) \leq c(n) \frac{a_{1}}{t} .
$$

But if $i_{0} \neq 1$, we have

$$
F_{\Omega}(x)=\int_{\Omega} \frac{1}{\left(\left(a_{i_{0}}+t-y_{i_{0}}\right)^{2}+\sum_{i \neq i_{0}} y_{i}^{2}\right)^{\frac{n}{2}}} d y .
$$


Replacing $y_{1}$ by 0 in the denominator, we see that

$$
F_{\Omega}(x) \leq 2 a_{1} \int_{\Omega^{\prime}} \frac{1}{\left(\left(a_{i_{0}}+t-y_{i_{0}}\right)^{2}+\sum^{\prime} y_{i}^{2}\right)^{\frac{n}{2}}} d y_{2} \cdots d y_{n},
$$

where $\Omega^{\prime}$ is the intersection of $\Omega$ with the coordinate hyperplane $y_{1}=0$, and $\sum^{\prime}$ means summation over $i \neq i_{0}, i \neq 1$. Proceeding as before, we substitute $s=a_{i_{0}}+t-y_{i_{0}}, u_{i}=\frac{y_{i}}{s}\left(i \neq i_{0}, i \neq 1\right)$ and extend integration with respect to $u_{i}$ over $(-\infty,+\infty)$. This leads to

$$
F_{\Omega}(x) \leq 2 a_{1} \int_{t}^{t+2 a_{i_{0}}} \frac{1}{s^{2}}\left(\int_{\mathbb{R}^{n-2}} \frac{1}{\left(1+\sum^{\prime} u_{i}^{2}\right)^{\frac{n}{2}}} d u\right) d s \leq c(n) \frac{a_{1}}{t} ;
$$

we have used (3.10) and (3.11). Inequalities (3.14) and (3.15) prove $a$ ).

Case $b$ ). Three possibilities may occur: $k<i_{0}, k=i_{0}$, and $k>i_{0}$. We treat them separately.

If $k<i_{0}$, we replace $y_{1}, \ldots, y_{k}$ by 0 and extend integration with respect to $y_{i}$ $\left(i>k, i \neq i_{0}\right)$ over $(-\infty,+\infty)$. Arguing much as in case $\left.a\right)$, we arrive at

$$
F_{\Omega}(x) \leq 2 a_{1} \cdot \ldots \cdot 2 a_{k} \int_{t}^{t+2 a_{i_{0}}} \frac{1}{s^{k+1}}\left(\int_{\mathbb{R}^{n-k-1}} \frac{1}{\left(1+\sum_{i} u_{i}^{2}\right)^{\frac{n}{2}}} d u\right) d s .
$$

Then (3.10) and (3.11) imply

$$
F_{\Omega}(x) \leq c(n) \frac{a_{1}}{t} \cdot \ldots \cdot \frac{a_{k}}{t} .
$$

If $k=i_{0}$, we replace $y_{1}, \ldots, y_{k-1}$ by 0 and extend integration in the variables $y_{k+1}, \ldots, y_{n}$ over $(-\infty,+\infty)$. After similar substitution we obtain

$$
F_{\Omega}(x) \leq 2 a_{1} \cdot \ldots \cdot 2 a_{k-1} \int_{t}^{t+2 a_{k}} \frac{1}{s^{k}}\left(\int_{\mathbb{R}^{n-k}} \frac{1}{\left(1+\sum_{i} u_{i}^{2}\right)^{\frac{n}{2}}} d u\right) d s .
$$

So, (3.9) and (3.11) imply

$$
F_{\Omega}(x) \leq c(n) \frac{a_{1}}{t} \cdot \ldots \cdot \frac{a_{k}}{t} .
$$

Finally, if $k>i_{0}$, we replace $y_{1}, \ldots, y_{i_{0}-1}, y_{i_{0}+1}, \ldots, y_{k}$ by 0 and extend integration in the variables $y_{k+1}, \ldots, y_{n}$ over $(-\infty,+\infty)$. Similar changes of variables yield

$$
\begin{gathered}
F_{\Omega}(x) \leq 2 a_{1} \cdot \ldots \cdot 2 a_{i_{0}-1} \cdot 2 a_{i_{0}+1} \cdot \ldots \cdot 2 a_{k} \\
\cdot \int_{t}^{t+2 a_{i_{0}}} \frac{1}{s^{k}}\left(\int_{\mathbb{R}^{n-k}} \frac{1}{\left(1+\sum u_{i}^{2}\right)^{\frac{n}{2}}} d u\right) d s \leq c(n) \frac{a_{1}}{t} \cdot \ldots \cdot \frac{a_{k}}{t} .
\end{gathered}
$$

We have used (3.9) and (3.11) at the last step. Now, b) follows from (3.16)(3.18).

Theorem 1 is now proved by an immediate application of Lemmas 1 and 2 . 


\section{Counterexample}

Examples given below show that the convexity condition in Theorem 1 is essential. We begin with the one-dimensional case; then we show how to use it for constructing an example on the plane with connected $\Omega$.

We will need the following simple observation.

Proposition 2. Let $\Omega$ be a bounded open subset of $\mathbb{R}^{n}$ such that the statement of Theorem 1 is correct. Then

$$
\left\|F_{\Omega}(x)\right\|_{L_{1, \infty}\left(\mathbb{R}^{n} \backslash \Omega\right)} \leq c|\Omega|,
$$

where $c=c(n)>0$ is a constant depending only on the dimension $n$.

Proof. Clearly,

$$
\varphi(t)=\sup _{x \in \mathbb{R}^{n} \backslash \Omega_{t}} F_{\Omega}(x)
$$

is a monotone nonincreasing function of $t$. Since $\Omega$ is bounded, the definition (1.1) of $F_{\Omega}$ implies that $\varphi(t) \rightarrow 0$ as $t \rightarrow \infty$. Since $\Omega$ is open, we have $\varphi(t) \rightarrow \infty$ as $t \rightarrow 0$. Next, the continuity of $F_{\Omega}$ shows that $\varphi$ has no jumps and, therefore, is continuous. So, $\varphi$ is a continuous monotone nondecreasing function taking all values between 0 and $\infty$. Now, take a number $c>0$ and put

$$
t_{c}=\inf \{t>0 \mid \varphi(t) \leq c\} .
$$

Then $\varphi\left(t_{c}\right)=c$, and if $F_{\Omega}(x)>c$, then $x \in \Omega_{t_{c}} \backslash \Omega$. Since we suppose that the statement of Theorem 1 is correct, we have

$$
\varphi\left(t_{c}\right) \leq c(n) \frac{|\Omega|}{\left|\Omega_{t_{c}} \backslash \Omega\right|},
$$

and therefore

$$
c \cdot\left|\left\{x \mid F_{\Omega}(x)>c\right\}\right| \leq \varphi\left(t_{c}\right) \cdot\left|\Omega_{t_{c}} \backslash \Omega\right| \leq c(n) \cdot|\Omega| .
$$

Remark 1. The inequality $\left\|F_{\Omega}(x)\right\|_{L_{1, \infty}\left(\mathbb{R}^{n} \backslash \Omega\right)} \leq c|\Omega|$ for a convex $\Omega$ with the constant dependent only on the dimension $n$ can also be obtained from the proof of Theorem 1 in [8] and some rather simple considerations connected with the convexity. Authors are very thankful to P. Sjögren for this observation.

Example 1. Consider an interval $(a, b) \subset \mathbb{R}$. We split it into $4 N$ subintervals of equal length and define $\Omega$ to be the union of even subintervals, that is, $\Omega=\bigcup_{k=1}^{2 N} I_{k}$, where

$$
I_{k}=\left(a+\frac{2 k-1}{4 N(b-a)}, a+\frac{2 k}{4 N(b-a)}\right) .
$$

Let $x \in(a, b) \backslash \Omega$. Then at least $N$ intervals $I_{k}$ lie to one side of $x$. Denote by $\Omega^{\prime}$ the union of these intervals. Then

$$
F_{\Omega}(x) \geq F_{\Omega^{\prime}}(x)=\sum_{I_{k} \in \Omega^{\prime}} F_{I_{k}}(x) .
$$


Therefore,

$$
\begin{gathered}
F_{\Omega}(x) \geq \sum_{k=1}^{N} \int_{\frac{2 k-1}{4 N(b-a)}}^{\frac{2 k}{4 N(b-a)}} \frac{1}{t} d t \geq \sum_{k=1}^{N} \frac{4 N(b-a)}{2 k} \cdot \frac{1}{4 N(b-a)} \\
=\sum_{k=1}^{N} \frac{1}{2 k} \geq \frac{1}{2} \sum_{k=1}^{N} \frac{1}{k} \geq c_{1} \ln \left(c_{2} N\right),
\end{gathered}
$$

where $c_{1}, c_{2}>0$ are universal constants. Since the measure of $(a, b) \backslash \Omega$ is $\frac{b-a}{2}$, we have

$$
\left|\left\{x \in \mathbb{R} \mid F_{\Omega}(x)>c_{1} \ln \left(c_{2} N\right)\right\}\right| \cdot c_{1} \ln \left(c_{2} N\right) \geq \frac{b-a}{2} \cdot c_{1} \ln \left(c_{2} N\right) .
$$

Remark 2. Since $N$ in (4.4) is arbitrary, this example already shows that, without the convexity assumption, the constant $c$ in Theorem 1 may become arbitrarily large.

Example 2. We continue the construction. Consider the union of the intervals $U_{k}=\left(\frac{1}{2^{k}}, \frac{1}{2^{k-1}}\right), k=1,2, \ldots$, and split each $U_{k}$ into $4 N_{k}$ subintervals of equal length. We denote by $\Omega_{k}$ the union of $2 N_{k}$ even intervals and put $\tilde{\Omega}=\bigcup \Omega_{k}$.

By (4.3), on the set $U_{k} \backslash \Omega_{k}$ we have

$$
F_{\Omega}(x) \geq c_{1} \ln \left(c_{2} N_{k}\right) .
$$

Since $\left|U_{k} \backslash \Omega_{k}\right|=\frac{1}{2^{k+1}}$, we see that

$$
\left|\left\{x \in \mathbb{R} \mid F_{\Omega}(x)>c_{1} \ln \left(c_{2} N_{k}\right)\right\}\right| \cdot c_{1} \ln \left(c_{2} N_{k}\right) \geq \frac{1}{2^{k+1}} \cdot c_{1} \ln \left(c_{2} N_{k}\right) .
$$

Therefore, if $N_{k}$ are chosen in such a way that the right-hand side tends to infinity as $k \rightarrow \infty$, then the claim of Theorem 1 fails for $\tilde{\Omega}$, whatever $c>0$.

The set $\tilde{\Omega} \subset \mathbb{R}$ constructed above is not connected; however, on the plane a connected set $\Omega$ with similar properties can be exhibited. For this, consider first the product $\tilde{\Omega} \times[0,1]$, where $\tilde{\Omega}$ was constructed above. Routine but somewhat lengthy calculations show that

$$
F_{\Omega}(x) \geq c_{1} \ln \left(c_{2} N_{k}\right)
$$

for $x \in\left(U_{k} \backslash \Omega_{k}\right) \times(0,1)$. Now, we add a "small cap" $[0,1] \times[1,1+\varepsilon]$, and take the interior of the set $(\tilde{\Omega} \times[0,1]) \cup([0,1] \times[1,1+\varepsilon])$ for $\Omega$. By (4.6), the claim of Corollary 1 and therefore of Theorem 1 fails for $\Omega$, whatever $c$. Clearly, $\Omega$ is open, connected and bounded.

The question now arises as to whether the claim of Theorem 1 is true for domains with $C^{\infty}$-smooth boundary. We show that in this case the constant $c$ may happen to be arbitrarily large. For this, we take the segment $[0,1]$, split it into $4 N$ subintervals of equal length, and denote by $\tilde{\Omega}$ the union of even subintervals. As is easily seen, the above construction (adding a "small cap") yields a set $\Omega \subset \mathbb{R}^{2}$ with rectifiable boundary, which can be regularized to become of class $C^{\infty}$. However, for this $\Omega$ the constant will be at least $c_{1} \ln \left(c_{2} N\right)$, where $c_{1}, c_{2}>0$ do not depend on $N$.

Thus, unlike the initial statement of Theorem 1, the constant can be made arbitrarily large. 


\section{REFERENCES}

1. B. Gustafsson and M. Putinar, An Exponential Transform and Regularity of Free Boundaries in Two Dimensions, Ann. Scuola Norm. Sup. Cl. Sci., 4, vol 26 (1998), pp. 507-543. MR1635702 (99k:30004)

2. B. Gustafsson and M. Putinar, The Exponential Transform: A Renormalized Riesz Potential at Critical Exponent, Indiana Univ. Math. J., 52 (2003), no. 3, pp.527-568. MR1986888 (2004c:31010)

3. B. Gustafsson, C. He, P. Milanfar and M. Putinar, Reconstructing Planar Domains from Their Moments, Inverse Problems, 16 (2000), no. 4, 1053-1070. MR1776483 (2001k:44010)

4. Handbook of Convex Geometry, Vol. B, North-Holland, Amsterdam, 1993.

5. N. Kruglyak and E.A. Kuznetsov, Smooth and Non-smooth Calderón-Zygmund Type Decompositions for Morrey Spaces, Journal of Fourier Analysis and Applications, 11, issue 6 (2005), 697-714. MR2190680(2006i:42026)

6. P. Mattila, Geometry of Sets and Measures in Euclidean Spaces, Cambridge Univ. Press, 1995, xii+343 pp. MR $1333890(96 \mathrm{~h}: 28006)$

7. M. Putinar, A Renormalized Riesz Potential and Applications, Advances in Constructive Approximation, Nashboro Press, Brentwood, TN, (2004), pp.433-465. MR2089943 (2005h:42041)

8. P. Sjögren, Weak $L_{1}$ Characterizations of Poisson Integrals, Green Potentials, and $H^{p}$ Spaces, Trans AMS, 233 (1977), 179-196. MR0463462 (57:3412)

9. E.M. Stein, Singular Integrals and Differentiability Properties of Functions, Princeton, New Jersey, 1975 (second printing with corrections), x+297 pp.

Department of Mathematics, Luleå University of Technology, 97187 Lule̊̊, Sweden E-mail address: natan@1tu.se

Department of Mathematics, Lule̊ University of Technology, 97187 Lule̊, Sweden

E-mail address: evgeny@sm.luth.se 